\title{
Análise Pedagógica e Ergonômica da Combinação de Diferentes Metodologias para o Ensino de Estrutura de Dados
}

\author{
André Luis Vieira Azevedo, UFPA, andre.azevedohist@gmail.com \\ Bruno Veloso dos Santos, UFPA, brvelosoo@gmail.com \\ Benedito Junior Almeida Leão, UFPA, junioleao75@gmail.com \\ Jacó Rodrigues de Alfaia, UFPA, alfaiajaco@gmail.com \\ Carlos dos Santos Portela, UFPA, csp@ufpa.br \\ Fabricio de Souza Farias, UFPA, fabriciosf@ufpa.br
}

\begin{abstract}
Resumo. Existem diversas metodologias que visam melhorar o desempenho dos alunos, no entanto, uma que se adequa para um estudante pode ser ineficiente para outro. Com isso o índice de desmotivação e desinteresse nas disciplinas de programação só aumenta. No curso de Sistemas de Informação da Universidade Federal do Pará isso é refletido em um elevado número de reprovações, que indica a necessidade da busca por novas metodologias para o ensino. Diante deste contexto, este trabalho visa avaliar o impacto pedagógico, ergonômico e de desempenho da aplicação de um conjunto de metodologias de ensino durante a disciplina de Estrutura de Dados I. Os resultados indicam que a metodologia proposta atende satisfatoriamente os critérios pedagógicos e ergonômicos, assim como, pode vir a auxiliar na redução do número de reprovações.
\end{abstract}

Palavras-Chave: metodologia, ensino-aprendizagem, ergonomia, pedagogia.

\section{Pedagogical and Ergonomic Analysis of the Combination of Different Methodologies for Data Structure Teaching}

\begin{abstract}
There are several methodologies that aim to improve student performance, however, one that is most suitable for one student may be inefficient for another. How it increases the rate of demotivation and disinterest in programming disciplines. In the information technology course, this is reflected in a high number of failures, which illustrates the need to search for new permanent learning methodologies. Given this context, this work aims to evaluate the pedagogical, ergonomic and performance impact of applying a set of teaching methods during a data structure I course. The results show that the proposed methodology follows the pedagogical and ergonomic criteria, as well as, reduces the number of failures.
\end{abstract}

Keywords: methodology, teaching-learning, ergonomics, pedagogy.

\section{Introdução}

$\mathrm{Na}$ área de ensino de computação e programação de computadores, diversos fatores podem resultar em desmotivação e evasão escolar dos alunos. Rigo et al. (2014) destacam como razões o elevado grau de exigência do mercado de trabalho, que cada vez mais busca um alto nível técnico por parte de novos profissionais, e a utilização de metodologias de ensino pouco atrativas em sala de aula. Além disso, Raabe, Zanchett e Vahldick (2015, p.1) ressaltam que a metodologia adotada no primeiro contato de alunos ingressantes no ensino superior, em conjunto com os principais conceitos abordados em programação de computadores, também são fatores determinantes sobre a forma como os alunos poderão vir a analisar os desafios apresentados em sua carreira e como poderão superar as dificuldades advindas da aprendizagem da lógica de programação. Deste modo, 
destacando a importância crucial na escolha da metodologia adotada em relação ao seu impacto na permanência ou desistência do discente no curso, assim como, o seu grau de interesse na área a partir deste contato.

De acordo com Twining et al. (2013) os professores em formação devem, essencialmente, conhecer diferentes ferramentas de apoio ao ensino e aprendizagem, como forma de integração inovadora da tecnologia para atender os estudantes do século 21. Pois a diversidade de uma turma impede que determinada metodologia unitária possa ser adequada para o atendimento por completo de todos os alunos. Por essa razão, Laburú, Arruda e Nardi (2003) ressaltam a importância da aplicação de um planejamento metodológico plural através do uso de meios e planejamentos pedagógicos diversos, que de acordo com os autores, são mais aconselháveis por respeitar a variedade de habilidades, preferências e tendências que estão presentes em uma turma. Na mesma perspectiva, Viveiro e Diniz (2009) exibem como vantagem fundamental o emprego da diversificação de ações e recursos didáticos, assim como a aplicação de estratégias para o encorajamento dos estudantes como um fator fundamental para a aprendizagem.

A partir deste contexto, torna-se necessário o enriquecimento do ambiente de ensino através da adoção de mais de uma metodologia de ensino e aprendizagem, assim possibilitando, pela diversificação do atendimento, o alcance ao maior número de alunos possíveis, isto é, respeitando a perspectiva de que os alunos possuem diferentes visões de mundo, formação familiar e história acadêmica.

Dentre as formas de diversificar o ambiente de aprendizagem de programação de computadores descritas na literatura, é possível destacar as seguintes abordagens: (1) o uso da tecnologia da informação, que por sua vez é uma alternativa para auxiliar professores e alunos; (2) a inserção de atividades lúdicas e motivacionais como a maratona de programação; e (3) a implantação de metodologias que fazem uso de jogos digitais em conjunto com a aprendizagem baseada em problemas (PBL).

Em relação à abordagem (1), Flôres e Tarouco (2008) enfatizam que os recursos baseados na tecnologia da informação possuem um grande potencial pedagógico, pois eles tornam as aulas mais atrativas e motivadoras e fazem a educação se beneficiar dos avanços da tecnologia. Baseado neste potencial pedagógico, Nichele e Schlemmer (2014) apresentam trabalhos que propõem e analisam diferentes conteúdos disponibilizados em diversas plataformas de ensino. Os resultados obtidos reforçam a importância de projetos que investiguem como melhorar a aprendizagem através de novas experiências interativas e colaborativas que envolvam as tecnologias digitais como alternativa presente no ambiente escolar.

No que diz respeito à abordagem (2), Herzberg, Mausner e Snyderman (1957) descrevem que a inserção de atividades que apresentam novos desafios aos estudantes na forma de competição é o que motiva muitos estudantes a aprimorar seu próprio esforço. Deste modo, a adoção de maratonas de programação pode ser um fator motivador para os indivíduos, pois apresentam problemas complexos que devem ser resolvidos durante um determinado período. Para Attle e Baker (2007), a oportunidade instrutiva de oferecer atividades de aprendizado em que os estudantes cooperam em times que competem uns com os outros maximiza o aprendizado dos alunos e seu desenvolvimento profissional, pois trata-se de um ambiente similar ao mundo corporativo.

Em relação a criação de jogos em ambientes escolares, abordagem de ensinoaprendizagem (3), Santos e Isotani (2018) afirmam que os jogos digitais, inseridos em ambientes escolares, usualmente contemplam atividades envolventes e atraentes, 
especialmente para os usuários mais jovens, o que pode trazer maior engajamento e aumentar a motivação dos estudantes. De maneira complementar, Santana, Araújo e Bittencourt (2018) destacam que os jogos exercem forte influência sobre a motivação dos estudantes e, assim, propõem melhorias nas experiências pedagógicas com o intuito de aumentar a participação e o engajamento dos estudantes em uma determinada disciplina. Com a aplicação dos jogos, os alunos podem abordar de forma interdisciplinar assuntos de diferentes disciplinas para a construção da solução de determinado problema que será abordado no ambiente virtual. Uma alternativa pedagógica para auxiliar a aplicação interdisciplinar dos assuntos é adoção da metodologia PBL, que por sua vez é baseada na participação ativa dos estudantes, onde o aluno passa a ter papel ativo no processo de ensino-aprendizagem, sendo responsável em discutir em grupos e encontrar soluções para os problemas apresentados durante as aulas de uma disciplina. Para Berbel (2011), com PBL os alunos que apresentam autonomia na realização de tarefas escolares se sentem mais à vontade a realizá-las e, com isso, seu desempenho será mais significativo. Já Ribeiro (2008) ressalta que a metodologia não é algo pronto a ser trabalhado, mas deve ser modelada de acordo com as necessidades e problematizações. Deste modo e em favor da aprendizagem dos alunos de uma turma, diferentes metodologias podem ser adotadas durante a mesma disciplina. Assim, aumentando as possibilidades de alcance dos alunos a partir de várias abordagens determinadas durante o planejamento pedagógico.

Diante deste contexto, este trabalho tem como objetivo avaliar o impacto pedagógico, ergonômico e de desempenho a partir da combinação de diferentes metodologias durante o processo de ensino-aprendizagem da disciplina de Estrutura de Dados I. Essa disciplina foi selecionada devido ser considerada uma das disciplinas com maiores índices de reprovação no curso de Sistemas de Informação da Universidade Federal do Pará (Oliveira et al., 2017) (Viana et al., 2019). Para condução do estudo, foram levadas em consideração a aula regular adotada em sala de aula e laboratório, as avaliações baseadas em maratona de programação visando estimular os alunos a melhorarem seus desempenhos e, por fim, a utilização da metodologia PBL e ensino dos conceitos em conjunto com a aplicação do desenvolvimento de jogos 2D.

O restante deste trabalho está organizado da seguinte forma: na Seção 2 são apresentados os conceitos relacionados à avaliação pedagógica e ergonômica, na Seção 3 é descrita a metodologia do estudo, na Seção 4 são apresentados os resultados obtidos e, por fim, na Seção 5 são relatadas as conclusões obtidas a partir do trabalho.

\section{Avaliação Pedagógica e Ergonômica}

A construção do conhecimento deve ser baseada na interação e discussão entre os alunos e professores, assim tornando a aquisição do conhecimento como o resultado da soma dos saberes de cada um durante todo o processo de construção (Hmelo-Silver, 2004). Para alcançar este objetivo uma metodologia de ensino-aprendizagem deve seguir critérios pedagógicos e ergonômicos.

Os critérios pedagógicos visam garantir que as metodologias adotadas para o ensino-aprendizagem estejam em conformidade com o objetivo educacional e com as necessidades do aprendiz. Já os critérios de análise ergonômica visam permitir ao usuário utilizar dos recursos de software adotados na prática de ensino considerando ao máximo a segurança, conforto e produtividade.

Nunes e Santos (2018) apresentam um estudo sobre à revisão das principais abordagens de avaliação de softwares educativos, e baseado nestas abordagens, realizam uma avaliação ergonômico-pedagógica de aplicativos relacionados às disciplinas 
abordadas no Exame Nacional do Ensino Médio (ENEM). Com isso levantando alguns questionamentos para expressar a importância da avaliação de softwares educativos, em virtude da necessidade de cuidados constantes dos profissionais da tecnologia e da educação e as competências necessárias para propagar informação de qualidade através da tecnologia digital. Esses cuidados, referem-se principalmente aos requisitos ergonômicos e pedagógicos que devem ser levados em consideração durante a adoção de metodologias de suporte ao ensino.

\section{Metodologia de Aplicação}

Enfrentar o desafio de minimizar o número de reprovações e evasão no curso de Sistemas de Informação na UFPA perpassa pela tarefa criativa de proposição de formas alternativas para avaliação do processo de ensino-aprendizagem. Deste modo, este trabalho avaliou durante a disciplina de Estrutura de Dados I o impacto pedagógico, ergonômico e de desempenho obtido a partir da participação dos alunos em um cenário composto por diferentes estímulos para a aprendizagem, isto é, (1) sala de aula e laboratório de computação, (2) com formas de avaliação baseadas na maratona de programação, e (3) com a utilização de jogos 2D e PBL como ferramentas de suporte.

Em (1), o ambiente acadêmico regular é composto por aula expositiva ministrada em um laboratório de informática contendo acesso à Internet e utilizando material didático em slides e códigos-fonte disponibilizados aos alunos na linguagem de programação C; Para (2), o método de avaliação é baseado em provas práticas, ou seja, no formato da maratona de programação organizada pela Sociedade Brasileira de Computação, que por sua vez organiza os alunos em grupos de três para a resolução dos problemas durante um tempo pré-determinado, assim como, recompensa cada equipe por acerto e, ao final, premia aquela com o melhor desempenho; e (3) corresponde ao atendimento dos alunos na forma de apresentação de novos conhecimentos que abordam os conteúdos de Estrutura de Dados I. Neste experimento os assuntos de fila e pilha foram apresentados de forma transversal através do ensino do desenvolvimento de jogos 2D utilizando a ferramenta Construct 2 para a construção de dois minigames, sendo o primeiro para a criação de um jogo de fila de banco e o segundo para a representação do problema clássico da torre de Hanói. A Figura 1(a) apresenta o minigame da fila de banco, enquanto que a Figura 1(b) ilustra o minigame baseado no problema da Torre de Hanói.

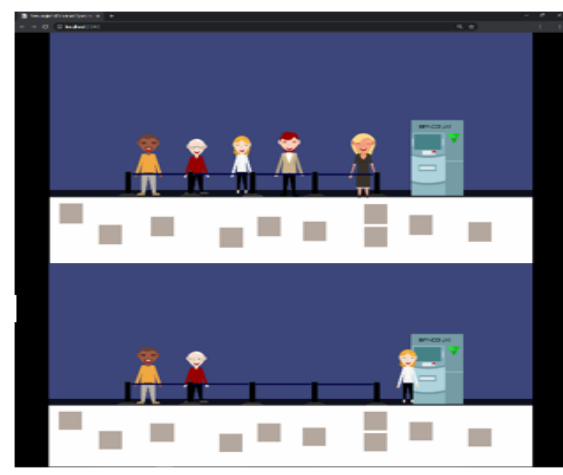

(a)

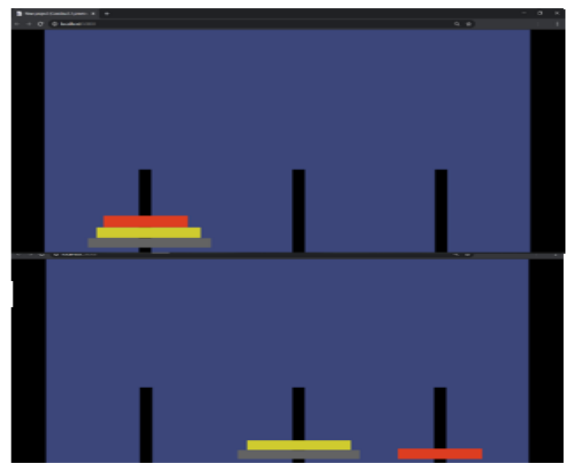

(b)

Figura 1: Imagens ilustrativas dos minigames da disciplina de Estrutura de Dados I. (a) Minigame sobre Filas. (b) Minigame sobre Pilha.

A condução da disciplina seguiu diferentes abordagens de ensino, isto é, considerando mais de um tipo de metodologia para atendimento dos alunos. Na primeira (1) foi levado em consideração a metodologia de ensino baseada em aula aberta e 
dialogada, na qual o aluno e professor contextualizam o problema e participam da construção de soluções teóricas e práticas viáveis para sua solução, sendo esta adotada pelo professor durante a carga horária de 44 horas da disciplina de Estrutura de Dados I.

Já na segunda (2) foi considerado a aprendizagem baseada na metodologia ativa de ensino, onde os estudantes foram desafiados a resolverem problemas práticos em equipe durante um tempo estipulado para a maratona de programação. A etapa de maratona de programação foi dividida em 4 partes, sendo 2 denominadas de simulados da maratona de programação e 2 denominadas de provas da maratona de programação. $O$ principal objetivo do simulado foi auxiliar os alunos na ambientação dos métodos de avaliação e nível das questões abordadas durante as maratonas de programação. A aplicação dos simulados e provas ocorreram aos sábados letivos com duração de 4 horas cada, isto é, totalizando 16 horas.

Por fim no terceiro (3), a aprendizagem baseada em problemas (PBL) foi escolhida como abordagem. Durante a aplicação do PBL, os alunos receberam um treinamento inicial sobre jogos 2D, Construct 2 e acerca dos assuntos de fila e pilha. Depois, equipes foram formadas e desafiadas a criar, de forma autônoma, dois jogos denominados de minigames, vide Figura 1. Durante a realização dessa atividade, os alunos participantes foram estimulados à discussão temática entre os membros de suas equipes e professores, assim possibilitando a criação de um ambiente técnico de discussão. A aplicação do PBL contou com uma carga horária de 8 horas, sendo divididas em 4 horas para o treinamento e 4 horas para a criação dos minigames. Com a conclusão de (3), contemplou-se as 68 horas-aula da disciplina de Estrutura de Dados I.

Para validar a metodologia de ensino proposta, ao final da disciplina de Estrutura de Dados I os alunos foram convidados a preencherem um formulário composto por perguntas que avaliam a disciplina e sua metodologia a partir de critérios pedagógicos e ergonômicos que devem ser levados em consideração para a garantia de uma adequada experiência de ensino-aprendizagem. Após o preenchimento, os resultados obtidos foram agrupados com os desempenhos obtidos pelos alunos participantes. A avaliação pedagógica e ergonômica utilizou como base a escala de Likert (1932), sendo representadas as opções de resposta por: (1) Discordo totalmente, (2) Discordo, (3) Não concordo nem discordo, (4) Concordo (5) Concordo plenamente.

Os critérios pedagógicos são baseados em Martínez-Rodriguez et al. (2019), que por sua vez salientam que a aplicação de novas metodologias de ensino e aprendizagem podem impactar positivamente na confiança dos estudantes e em seus hábitos de estudo fora da sala de aula, tornando, assim também necessário avaliar a capacidade de motivação, generalização para resolução de problemas parecidos, escolha entre o ensino tradicional e metodologia proposta, capacidade de trabalhar em equipe e comprometimento do aluno com as atividades propostas. Já os critérios ergonômicos são baseados em termos conceituais, isto é, um conjunto de conhecimentos científicos relativos ao homem e necessários para a geração de instrumentos e dispositivos que o mesmo possua segurança e conforto (Hack et al., 1999). Com isso, os critérios avaliam os impactos: da avaliação em grupo e baseada na maratona de programação; da metodologia no rendimento dos alunos; da avaliação na forma de maratona de programação; do papel do professor no sucesso dos alunos; da qualidade da aula de ensino de jogos; e da forma de repasse dos conteúdos abordados durante as aulas.

Por fim, para avaliação do desempenho dos alunos foi levado em consideração através da adoção dos critérios estabelecidos por Nunes e Santos (2018), os quais destacam que o uso de softwares voltados para o ensino-aprendizagem dentro da sala de 
aula pode servir como auxílio para os alunos, visto que ajuda os mesmos na fixação e no melhor aproveitamento do conteúdo que foi repassado durante a aula. Deste modo, o relacionamento da educação com a tecnologia pode enriquecer a qualidade do processo de ensino-aprendizagem, o que causa impacto direto nos métodos, avaliando o desempenho em relação: ao impacto da metodologia; a participação dos membros do grupo; a dedicação dos participantes na realização da tarefa; ao rendimento obtido a partir das novas metodologias baseadas em jogos 2D e maratona de programação; e ao impacto de uma avaliação baseada nas etapas de simulado e prova.

\section{Resultados}

A Tabela 1 apresenta os resultados da análise pedagógica. São considerados os seguintes resultados da análise: (PP1) se os alunos se sentiram motivados para realização das tarefas; (PP2) se tiveram a certeza em resolver problemas parecidos; (PP3) se preferem as aulas tradicionais em detrimento das aulas que envolvem o uso de jogos $2 \mathrm{D}$ ou maratona de programação; (PP4) se os alunos preferem o método de avaliação tradicional baseado em provas individuais; e (PP5) se dedicaram total atenção a disciplina. A partir dos resultados, é possível identificar percentuais positivos de concordância e concordância total para as afirmativas PP1, PP2 e PP5 obtendo os respectivos índices, 90,9\%,68.2\% e 63,7\%. De maneira geral, é possível identificar um percentual positivo superior a $75 \%$ para as três respostas. Além disso, observa-se que a menor nota foi atribuída à dedicação dos alunos em relação a participação e realização das atividades.

Tabela 1 - Resultados da Visão Pedagógica dos Alunos da Disciplina Estrutura de Dados I.

\begin{tabular}{|c|l|c|c|c|c|c|}
\hline PP\# & \multicolumn{1}{|c|}{ PERGUNTAS PEDAGÓGICAS } & 1 & 2 & 3 & 4 & 5 \\
\hline PP1 & $\begin{array}{l}\text { Nas avaliações com premiação me } \\
\text { motivaram a ter uma persistência pra } \\
\text { realização final das tarefas. }\end{array}$ & $0 \%$ & $0 \%$ & $9,1 \%$ & $63,6 \%$ & $27,3 \%$ \\
\hline PP2 & $\begin{array}{l}\text { Depois dessa disciplina, tenho a certeza que } \\
\text { poderei resolver problemas parecidos }\end{array}$ & $0 \%$ & $4,5 \%$ & $27,3 \%$ & $50 \%$ & $18,2 \%$ \\
\hline PP3 & $\begin{array}{l}\text { De acordo com as experiências anteriores } \\
\text { em sala de aula, preferi as aulas } \\
\text { tradicionais, simples e intuitivas que não } \\
\text { utilizam jogos 2D, ou premiação, ou } \\
\text { trabalho em equipe durante as avaliações. }\end{array}$ & $27,3 \%$ & $40,9 \%$ & $18,2 \%$ & $9,1 \%$ & $4,5 \%$ \\
\hline PP4 & $\begin{array}{l}\text { Seria possível obter melhores resultados } \\
\text { nas avaliações, caso as mesmas fossem } \\
\text { aplicadas de forma individual. }\end{array}$ & $13,6 \%$ & $59,1 \%$ & $18,2 \%$ & $9,1 \%$ & $0 \%$ \\
\hline PP5 & $\begin{array}{l}\text { Estrutura de Dados I. } \\
\text { Dediquei atenc̃ão total à disciplina }\end{array}$ & $0 \%$ & $0 \%$ & $36,3 \%$ & $45,5 \%$ & $18,2 \%$ \\
\hline
\end{tabular}

Em relação as perguntas PP3 e PP4, os resultados indicam que a maioria dos participantes discordam ou discordam totalmente que o método de ensino tradicional e a avaliação individual poderiam retornar melhores resultados do que o alcançado com a 
metodologia composta por mais métodos, sendo obtidos os respectivos percentuais de discordância, $68,2 \%$ e $72,7 \%$.

A Tabela 2 apresenta os resultados da análise ergonômica, cuja as perguntas de avaliação foram: (PE1) se a dinâmica adotada na disciplina teve papel crucial na realização das tarefas; (PE2) se negaram que o método tradicional é mais eficiente; (PE3) se concordaram que as maratonas ajudaram nas realizações das tarefas; (PE4) se os alunos obtiveram atenção total durante a aplicação da metodologia dos jogos 2D; (PE5) se o minicurso de jogo 2D foi objetivo e claro; e se (PE6) o conteúdo repassado pelo professor não deixou dúvidas. A partir dos resultados, é possível identificar percentuais positivos de concordância e concordância total para as afirmativas PE1, PE3, PE4, PE5 e PE6 obtendo os respectivos índices de concordância 95,5\%, 90,9\%, 81,9\%, 77,2\%, 90,9\%. Já para a pergunta PE2, 68,2\% dos alunos discordam que o resultado poderia ser melhor com o ensino tradicional. Deste modo, os resultados indicam uma ergonomia positiva em relação ao uso de jogos 2D e maratona de programação. Deste modo, refletindo os resultados de Sales, Clímaco e Sales (2016) que destacam a importância de adotar novas metodologias de ensino, assim trazendo as características de jogos 2D que extrapolam o entretenimento, mas mantendo o caráter lúdico.

Tabela 2 - Resultados da Visão Ergonômica da Disciplina Estrutura de Dados I.

\begin{tabular}{|c|c|c|c|c|c|c|}
\hline PE\# & PERGUNTAS ERGONÔMICAS & 1 & 2 & 3 & 4 & 5 \\
\hline PE1 & $\begin{array}{l}\text { A dinâmica utilizada nas avaliações, } \\
\text { estilo maratona com grupo de três, foi } \\
\text { de total relevância para realização da } \\
\text { tarefa }\end{array}$ & $0 \%$ & $0 \%$ & $4,5 \%$ & $27,3 \%$ & $68,2 \%$ \\
\hline PE2 & $\begin{array}{l}\text { De acordo com as experiências } \\
\text { anteriores em sala de aula, eu obteria um } \\
\text { rendimento melhor caso as aulas } \\
\text { tivessem sido ministradas seguindo o } \\
\text { modo tradicionais baseado em } \\
\text { explanação do professor, listas de } \\
\text { exercícios e provas individuais }\end{array}$ & $27,3 \%$ & $40,9 \%$ & $18,2 \%$ & $9,1 \%$ & $4,5 \%$ \\
\hline PE3 & $\begin{array}{l}\text { A avaliação em forma de maratona, foi } \\
\text { de fundamental importância para } \\
\text { realizações das tarefas. }\end{array}$ & $0 \%$ & $0 \%$ & $9,1 \%$ & $54,5 \%$ & $36.4 \%$ \\
\hline PE4 & $\begin{array}{l}\text { Tive a atenção necessária dos } \\
\text { ministrantes do minicurso de jogos para } \\
\text { conseguir alcançar o meu objetivo. }\end{array}$ & $0 \%$ & $0 \%$ & $18,2 \%$ & $45,5 \%$ & $36,3 \%$ \\
\hline PE5 & $\begin{array}{l}\text { A aula do minicurso de jogos foi } \\
\text { ministrada de forma a não deixar } \\
\text { dúvidas sobre o que eu estava } \\
\text { aprendendo. }\end{array}$ & $0 \%$ & $0 \%$ & $22,7 \%$ & $50 \%$ & $27,3 \%$ \\
\hline PE6 & $\begin{array}{l}\text { O conteúdo repassado pelo professor } \\
\text { em sala foi objetivo e claro. }\end{array}$ & $0 \%$ & $0 \%$ & $9,1 \%$ & $54,5 \%$ & $36,4 \%$ \\
\hline
\end{tabular}

A Tabela 3 apresenta os resultados do desempenho dos alunos, sendo analisados: (DA1) se sentiram motivação em aprender mais ao final da disciplina; (DA2) se o trabalho 
em equipe foi de grande importância; (DA3) se os alunos se dedicaram totalmente na disciplina; (DA4) se obtiveram um rendimento melhor com o minigame e a maratona; e (DA5) se os erros cometidos motivaram a ter um desempenho maior. A partir dos resultados, é possível identificar percentuais positivos de concordância e concordância total para as afirmativas DA1, DA2, DA3, DA4 e DA5 obtendo os respectivos índices de concordância, $72,3 \%, 77,3 \%, 63,7 \%, 81,8 \%$ e $91 \%$. A partir da percepção dos alunos, a metodologia composta por vários métodos foi responsável pela melhoria em seu desempenho durante a disciplina de Estrutura de Dados I, assim, demonstrando a eficácia da metodologia proposta a partir da visão do estudante.

Tabela 3 - Resultados da Visão sobre o Desenvolvimento do Aluno na Disciplina Estrutura de Dados I.

\begin{tabular}{|c|c|c|c|c|c|c|}
\hline DA\# & DESEMPENHO DOS ALUNOS & 1 & 2 & 3 & 4 & 5 \\
\hline DA1 & $\begin{array}{l}\text { Com o minicurso de jogos e a abordagem de } \\
\text { avaliação baseada em maratona de } \\
\text { programação, me motivei a persistir e querer } \\
\text { aprender mais após o encerramento da } \\
\text { disciplina }\end{array}$ & $0 \%$ & $9,1 \%$ & $18,2 \%$ & $46.4 \%$ & $26,3 \%$ \\
\hline DA2 & $\begin{array}{l}\text { Todos os membros do seu grupo se } \\
\text { empenharam em ajudar para realização das } \\
\text { tarefas propostas durante as avaliações. }\end{array}$ & $0 \%$ & $0 \%$ & $22,7 \%$ & $36,4 \%$ & $40,9 \%$ \\
\hline DA3 & $\begin{array}{l}\text { Dediquei atenção total à disciplina Estrutura de } \\
\text { Dados I. }\end{array}$ & $0 \%$ & $0 \%$ & $36,3 \%$ & $45,5 \%$ & $18,2 \%$ \\
\hline DA4 & $\begin{array}{l}\text { Obtive um rendimento melhor com a realização } \\
\text { dos minicursos de jogos e os simulados com } \\
\text { premiação. }\end{array}$ & $0 \%$ & $0 \%$ & $18,2 \%$ & $63,6 \%$ & $18.2 \%$ \\
\hline DA5 & $\begin{array}{l}\text { Os erros que eu cometi na realização dos } \\
\text { simulados me motivaram a estudar mais para } \\
\text { ter um melhor rendimento nas provas }\end{array}$ & $0 \%$ & $4,5 \%$ & $4.5 \%$ & $81,9 \%$ & $9,1 \%$ \\
\hline
\end{tabular}

Outra análise pode ser feita a partir da comparação do índice de reprovações obtido a partir da aplicação da metodologia composta em relação à média de reprovações nos anos anteriores. A Figura 2 apresenta o gráfico que compara a porcentagem de reprovações entre os anos 2011 a 2018, que era de 44,88\%, com a média obtida em 2019. Sendo positiva a aplicação de mais de uma metodologia para adaptação do aluno, pois obteve-se média de reprovação igual a 10,34\%. Ambas as médias foram extraídas dos relatórios do Sistema Integrado de Gestão de Atividades Acadêmicas (SIGAA) da UFPA.

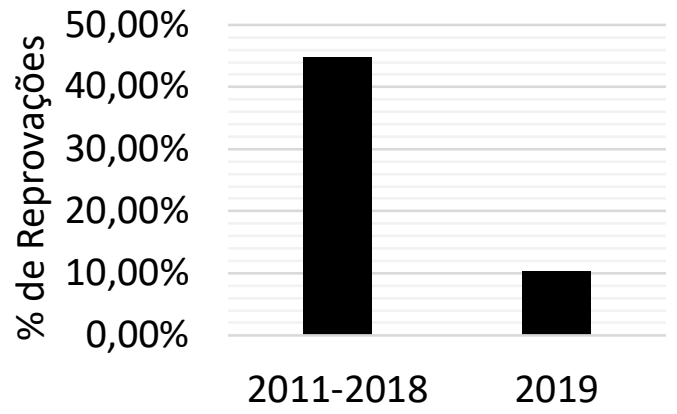

Figura 2: Médias de reprovação dos alunos de Estrutura de Dados I.

\section{Conclusão}


Este trabalho teve como objetivo avaliar o impacto pedagógico, ergonômico e de desempenho da utilização de um conjunto de metodologias de ensino durante a disciplina de Estrutura de Dados I do curso de Sistemas de Informação da Universidade Federal do Pará. Para avaliar a aplicação em sala de aula, foi levado em consideração o desempenho dos alunos através de um questionário que avaliava as questões ergonômicas, pedagógicas e de desempenho. A partir da análise dos resultados obtidos, pode-se concluir que o método proposto é capaz de auxiliar no processo de ensino-aprendizagem, pois permitiu aumentar o engajamento dos alunos e o interesse por parte deles na disciplina.

Este estudo indica que a proposta segue os critérios pedagógicos necessários para a aplicação de um conjunto de diferentes metodologias em sala de aula. Deste modo, os resultados demonstram um nível médio de aceitação considerado por $73 \%$ dos alunos. Em relação ao quesito ergonomia o índice médio geral de concordância com a maratona de programação, desenvolvimento de jogos 2D foi de $80 \%$. Por fim, a partir dos índices obtidos, observou-se a redução no número de reprovações na disciplina Estrutura de Dados I em 34,54\%. Neste sentido, acredita-se que a metodologia proposta pode vim a ajudar alunos e professores no ensino-aprendizagem de disciplinas na área de programação.

A partir desta pesquisa, reforça-se o fato de que professores devem buscar pela diversificação de sua metodologia, assim alcançando mais estudantes a partir do oferecimento de mais de uma abordagem metodológica de ensino. Com este trabalho, pode-se concluir que a aplicação de um conjunto de metodologias pode permitir ao professor alcançar um maior número de alunos, assim como, pode resultar na redução do número de reprovações e no aumento do nível de satisfação dos alunos em relação aos métodos de ensino adotados. Por fim, observa-se a oportunidade de se realizar uma pesquisa do tipo estudo de caso, para avaliar a aplicação desta combinação de abordagens de ensino em outras disciplinas da área de computação, e por outros docentes e alunos, a fim de analisar se os resultados obtidos serão semelhantes aos relatados neste artigo.

\section{Referências}

ATTLE, S.; BAKER, B. Cooperative learning in a competitive environment: classroom applications. International Journal of Teaching and Learning in Higher Education, 19(1):77-83, 2007.

BERBEL, N. As metodologias ativas e a promoção da autonomia de estudantes. Semina: Ciências Sociais e Humanas, v. 32, n. 1, p. 25-40, 2011.

FLÔRES. M.; TAROUCO. L. Diferentes tipos de objetos para dar suporte a aprendizagem. Em Revista Novas Tecnologias na Educação (RENOTE), V. 6 N $^{\circ}$, Julho, 2008.

HACK, C.; SOUZA, C.; THOMÉ, A.; CORRÊA, Z.; ABREU, C. Ergonomia em Software Educacional: A Possível Integração entre Usabilidade e Aprendizagem. In: ATAS IHC 99, São Paulo, 1999.

HERZBERG, F.; MAUSNER, B.; SNYDERMAN, B. Job attitudes: Review of research and opinion. Psychological Service, 1957.

HMELO-SILVER, C. Problem-based learning: What and how do students learn?. Educational psychology review, v. 16, n. 3, p. 235-266, 2004.

LABURÚ, C.; ARRUDA, S.; NARDI, R. Pluralismo Metodológico no Ensino de Ciências. Ciência \& Educação, v. 9, n. 2, p. 247-260, 2003. 
LIKERT, R. A Technique for the Measurement of Attitudes. Archives of Psychology, 1932.

MARTÍNEZ-RODRÍGUEZ， R.; ALVAREZ-XOCHIHUA， O.; VICTORIA， O.; ARÁMBURO, A.; FRAGA, J. Use of Machine Learning to Measure the Influence of Behavioral and Personality Factors on Academic Performance of Higher Education Students. IEEE Latin America Transactions, v. 17, n. 04, p. 633-641, 2019.

NICHELE, A.; SCHLEMMER, E. Aplicativos para o ensino e aprendizagem de química. Em Revista Novas Tecnologias na Educação (RENOTE), v. 12, n. 2, 2014.

NUNES, R.; SANTOS, I. A Importância da Avaliação Ergonômico-Pedagógica de Aplicativos Educacionais e os Desafios Encontrados no Campo da Aprendizagem Móvel. In: CONGRESSO BRASILEIRO DE INFORMÁTICA NA EDUCAÇÃO (CBIE), Fortaleza, 2018, p. 41-50.

OLIVEIRA, N.; FARIAS, F.; MOURA, D.; ANJOS, J. Aprendendo Lógica Computacional Usando o Aplicativo AlgoFácil In: CONGRESSO DE TECNOLOGIAS E DESENVOLVIMENTO NA AMAZÔNIA (CTDA), Cametá, 2017.

RAABE, A.; ZANCHETT, G.; VAHLDICK, A. Jogos de Programar como uma Abordagem para os Primeiros Contatos dos Estudantes com à Programação. In: CONGRESSO BRASILEIRO DE INFORMÁTICA NA EDUCAÇÃO (CBIE), Maceió, 2015, p. 1485-1494.

RIBEIRO, L. Aprendizagem baseada em problemas (PBL) na educação em engenharia. Revista de Ensino de Engenharia, v. 27, n. 2, p. 23-32, 2008.

RIGO, S.; CAMBRUZZI, W.; BARBOSA, J.; CAZELLA, S. Aplicações de Mineração de Dados Educacionais e Learning Analytics com foco na evasão escolar: oportunidades e desafios. Em Revista Brasileira de Informática na Educação, v. 22, n. 01, p. 132146, 2014.

SALES, A.; CLÍMACO, G.; SALES M. Jogos Sérios em Interação HumanoComputador: Uma revisão Sistemática de Literatura. Em Revista Novas Tecnologias na Educação (RENOTE), v.14, n.1, 2016.

SANTANA, B.; ARAÚJO, L.; BITTENCOURT, R. Considerando a Motivação dos Estudantes em Experiências de Ensino-Aprendizagem de Computação. In: CONGRESSO BRASILEIRO DE INFORMÁTICA NA EDUCAÇÃO (CBIE), Fortaleza, 2018, p.500-509.

SANTOS, W.; ISOTANI, S. Desenvolvimento de Jogos Educativos? Desafios, Oportunidades e Direcionamentos de Pesquisa. Em Revista Novas Tecnologias na Educação (RENOTE), v.16, n.2, 2018.

TWINING, P; RAFFAGHELLI, J.; ALBION, P.; KNEZEK, D. Moving Education into the Digital Age: The contribution of Teachers' Professional Development. Journal of Computer Assisted E-Learning, Special Issue: Research-informed Strategies for Moving Education into the Digital Age - Outcomes of the International Summit on ICT in Education, 29 (5), 426-437, October. 2013.

VIANA, G.; LOPES, A.; PORTELA, C.; OLIVEIRA, S. Um Survey sobre a Aprendizagem de Programação no Curso de Sistemas de Informação. In: WORKSHOP SOBRE EDUCAÇÃO EM COMPUTAÇÃO (WEI), Belém, 2019, p. 161-175.

VIVEIRO, A.; DINIZ, R. Atividades de campo no ensino das ciências e na educação ambiental: refletindo sobre as potencialidades desta estratégia na prática escolar. Ciência em Tela, v.2, n.1, 2009. 